\title{
A Bayesian Framework to Constrain the Photon Mass with a Catalog of Fast Radio Bursts
}

\author{
Lijing Shao ${ }^{1, *}$ and Bing Zhang ${ }^{2,3,4, \dagger}$ \\ ${ }^{1}$ Max Planck Institute for Gravitational Physics (Albert Einstein Institute), Am Mühlenberg 1, Potsdam D-14476, Germany \\ ${ }^{2}$ Department of Physics and Astronomy, University of Nevada, Las Vegas, NV 89154, USA \\ ${ }^{3}$ Kavli Institute for Astronomy and Astrophysics, Peking University, Beijing 100871, China \\ ${ }^{4}$ Department of Astronomy, School of Physics, Peking University, Beijing 100871, China
}

\begin{abstract}
A hypothetical photon mass, $m_{\gamma}$, gives an energy-dependent light speed in a Lorentz-invariant theory. Such a modification causes an additional time delay between photons of different energies when they travel through a fixed distance. Fast radio bursts (FRBs), with their short time duration and cosmological propagation distance, are excellent astrophysical objects to constrain $m_{\gamma}$. Here for the first time we develop a Bayesian framework to study this problem with a catalog of FRBs. Those FRBs with and without redshift measurement are both useful in this framework, and can be combined in a Bayesian way. A catalog of 21 FRBs (including 20 FRBs without redshift measurement, and one, FRB 121102, with a measured redshift $z=0.19273 \pm 0.00008$ ) give a combined limit $m_{\gamma} \leq 8.7 \times 10^{-51} \mathrm{~kg}$, or equivalently $m_{\gamma} \leq 4.9 \times 10^{-15} \mathrm{eV} / c^{2}\left(m_{\gamma} \leq 1.5 \times 10^{-50} \mathrm{~kg}\right.$, or equivalently $m_{\gamma} \leq 8.4 \times 10^{-15} \mathrm{eV} / c^{2}$ ) at $68 \%(95 \%)$ confidence level, which represents the best limit that comes purely from kinematics. The framework proposed here will be valuable when FRBs are observed daily in the future. Increment in the number of FRBs, and refinement in the knowledge about the electron distributions in the Milky Way, the host galaxies of FRBs, and the intergalactic median, will further tighten the constraint.
\end{abstract}

PACS numbers: $14.70 . \mathrm{Bh}, 41.20 . \mathrm{Jb}, 52.25 . \mathrm{Os}, 95.85 . \mathrm{Bh}$

\section{INTRODUCTION}

The special relativity postulates the "constancy of light speed" as a fundamental principle in physics [1]. It is extended into the general relativity and quantum field theories. In quantum mechanics, the particle-wave duality translates the constancy of light speed into the "masslessness of photons" [2]. Nevertheless, there exist theories with massive photons. The Maxwell-de Broglie-Proca theory is a famous example where photons obtain mass in the cost of the gauge invariance [3, 4]. Another example where "effectively massive photons" arise due to a possible oscillation between the canonical U(1) photons and hypothetical U'(1) "photons" [5]. Despite the celebrated success of the postulate, these scenarios are interesting and worthy to investigate, whereas the ultimate word on the photon mass roots in empirical facts.

Great efforts were put to various kinds of experiment to push the empirical boundary on the "masslessness of photons" $[6,7]$. These tests start from early experiment in testing the $\propto r^{-2}$ Coulomb law [8], to Schumann resonance in cavity [9], gravitational deflection of electromagnetic waves [10], mechanical stability of magnetized gas in galaxies [11], Jupiter's magnetic field [12], toroid Cavendish balance [13, 14], magneto-hydrodynamics of the solar wind [15, 16], black hole bombs [17], and spindown of a white-dwarf pulsar [18]. These tests involve the anomalous dynamics introduced by the mass term of photons, while there exist cleaner tests which only involve the anomalous kinematics introduced by the mass [19-22], thus independent of the underlying massive photon theory. The latter kind of tests use the propagation of photons through a cosmological distance, and

\footnotetext{
*lijing.shao@aei.mpg.de

$\dagger$ zhang@physics.unlv.edu
}

examine the time delay between photons with different energies. In this paper we will study the empirical mass constraint on the photon mass from the propagation of electromagnetic waves of fast radio bursts (FRBs) [23-25].

If photon has a mass $m_{\gamma}$, the Lorentz-invariant dispersion relation reads,

$$
E^{2}=p^{2} c_{0}^{2}+m_{\gamma}^{2} c_{0}^{4}
$$

where $c_{0}$ is the limiting velocity for high energy photons. The group velocity for a photon with energy $E=h v$ is,

$$
v \equiv \frac{\partial E}{\partial p}=\frac{p c_{0}}{E} c_{0} \simeq c_{0}\left[1-\frac{1}{2}\left(\frac{m_{\gamma} c_{0}^{2}}{h v}\right)^{2}\right],
$$

where the last derivation holds when $m_{\gamma} \ll h v / c_{0}^{2} \simeq 7 \times$ $10^{-42} \mathrm{~kg}\left(\frac{v}{\mathrm{GHz}}\right)$. It is evidently clear from Eq. (2) that when the energy of a photon is smaller, the relative modification is larger. ${ }^{1}$ Because we here study the test that involves the accumulative time delay from light propagation, it is easy to understand that (i) finer time structure of the event, (ii) longer propagation distance, and (iii) lower energy for photons, define the figure of merit of the test. With this setting, FRBs provide a superb celestial laboratory for testing the photon mass, because -

1. they are very short and even not temporally resolved by the receivers in general, and

2. they are believed to be cosmological objects with nonnegligible redshifts, and

3. they are observed at radio frequency, which leverages the smallness of $m_{\gamma}$ in Eq. (2).

\footnotetext{
${ }^{1}$ This is opposite to the test of Lorentz invariance violation with light propagation [26-28], where high energy photons are preferred to put constraints.
} 
The first work using FRBs to constrain the photon mass, performed by $\mathrm{Wu}$ et al. [20] and Bonetti et al. [21], used FRB 150418 [29]. However, the measurement of the redshift for this FRB was challenged with a flare in a radio-variable active galactic nucleus [30,31], and now the measurement is generally believed to be unreliable [32]. A reliable measurement of the redshift was made for FRB 121102 [32, 33], and Bonetti et al. [22] followed up the measurement to constrain the photon mass to be $m_{\gamma} \leq 3.9 \times 10^{-50} \mathrm{~kg}$, or equivalently, $m_{\gamma} \leq 2.2 \times 10^{-14} \mathrm{eV} / c^{2}$. The method proposed in these papers needs a measurement of the redshift for FRBs, however, up to now, only one FRB is fortunate enough to identify the host galaxy and gets a redshift measurement. Since the localization of an FRB is facilitated if the source is repeating and since none of the other FRBs are observed to repeat so far, the sample of redshift-measured FRBs may not grow significantly in the near future [34]. Therefore, we here extend these work to FRBs where the redshift is not available. We construct a Bayesian formula to derive a combined constraint from a catalog of FRBs, where uninformative prior is made to the redshift. Figure 1 shows the sky distribution of FRBs that are used in this work (see also Table I) [35].

The paper is organized as follows. In the next section, the theoretical framework for data analysis is laid which includes a hypothesis for the $v^{-2}$-behaved time delay, and a Bayesian framework to constrain $m_{\gamma}$. In Section III we examine our uninformative treatment of the redshift with FRB 121102, and present the constraint that comes from a combination of a catalog of 21 FRBs where only one of them has a redshift measurement. Section IV summarizes the work and discusses future prospects in constraining the photon mass with FRBs.

\section{THEORETICAL FRAMEWORK}

We review a hypothesis on the $v^{-2}$ behavior observed in the time delay of FRBs in Section II A, and then construct a Bayesian framework in Section II B to analyze the observed FRB data.

\section{A. A hypothesis on the time delay}

Here we present the hypothesis of time delay for FRBs when photon has a mass, $m_{\gamma}$ [20-22]. The hypothesis, $\mathcal{H}$, will be used in the Bayesian inference in Section II B.

From observations, all FRBs show an indisputable $v^{-2}$ dependent time delay, $\Delta t \propto v^{-2}[35,45]$. In our hypothesis, we attribute the delay to two causes, (i) the propagation of electromagnetic wave through ionized median, and (ii) the mass term of photon. Some remarks come as follows -

- The interaction between the propagating electromagnetic wave and the ionized median introduces a time delay, $\Delta t_{\mathrm{DM}}$, for a photon with energy, $E=h v$, relative to a photon with an infinite energy [46],

$$
\Delta t_{\mathrm{DM}}=\int \frac{\mathrm{d} l}{c_{0}} \frac{v_{\mathrm{p}}^{2}}{2 v^{2}} \simeq 4.15 \mathrm{~ms}\left(\frac{\mathrm{DM}_{\mathrm{astro}}}{\mathrm{pc} \mathrm{cm}^{-3}}\right)\left(\frac{v}{1 \mathrm{GHz}}\right)^{-2},
$$
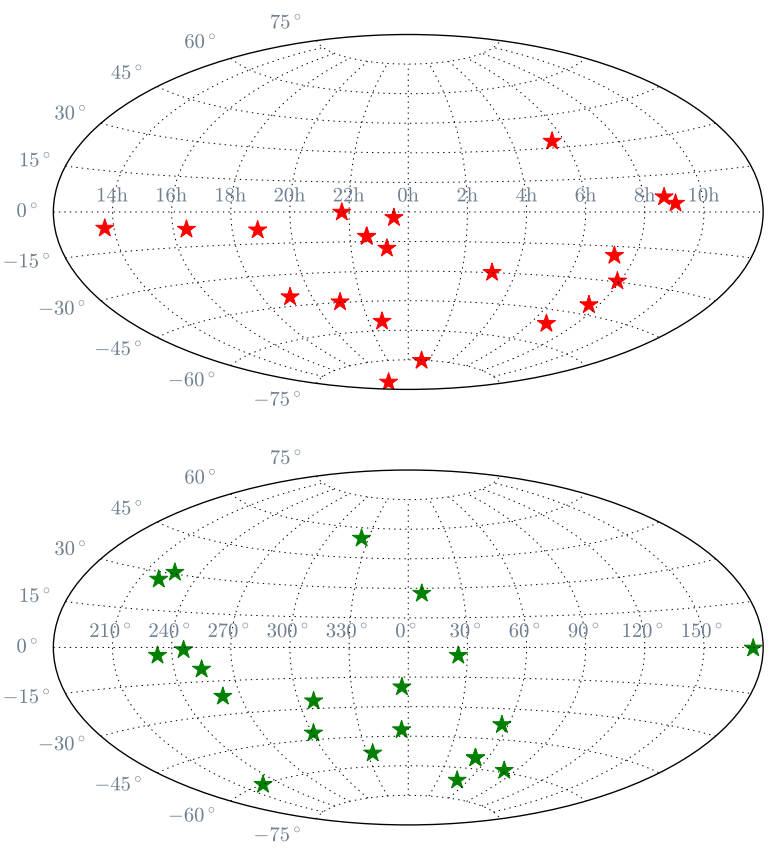

FIG. 1. Distribution of FRBs that are used in constraining the photon mass [35] in (upper) celestial coordinate, and (lower) galactic coordinate.

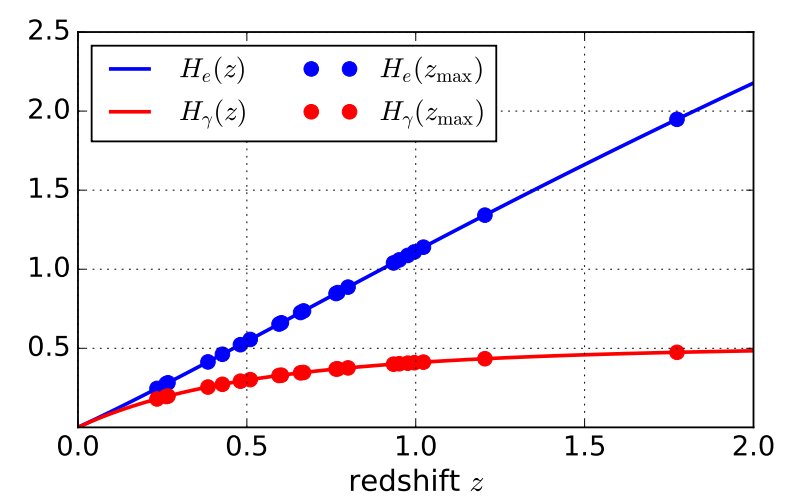

FIG. 2. The dependence of functions $H_{e}(z)$ and $H_{\gamma}(z)$ on the redshift. Their function values evaluated at the maximum redshift, $z_{\max }$, for 21 FRBs in Table I, are shown with circles.

where the plasma frequency $v_{\mathrm{p}} \equiv \sqrt{n_{e} e^{2} / 4 \pi^{2} m_{e} \epsilon_{0}}$ with $n_{e}$ the number density of electrons, $e$ the charge of an electron, $m_{e}$ the mass of an electron, and $\epsilon_{0}$ the permittivity of free space. The dispersion measure is defined as the integral of the electron number density along the path, $\mathrm{DM}_{\text {astro }} \equiv \int n_{e} \mathrm{~d} l$. In a cosmological setting, $\mathrm{DM}_{\text {astro }} \equiv \int(1+z)^{-1} n_{e}^{(0)} \mathrm{d} l$ where $z$ is the redshift and $n_{e}^{(0)}$ is the electron number density in the rest frame [47].

In Eq. (3), different sources contribute to the dispersion measure, $\mathrm{DM}_{\text {astro }}$ [20-22, 35], notably from the Milky Way, $\mathrm{DM}_{\mathrm{MW}}$, from the intergalactic median (IGM), $\mathrm{DM}_{\mathrm{IGM}}$, and from the host galaxy, $\mathrm{DM}_{\text {host }}$. Therefore, 
TABLE I. A catalog of FRBs ${ }^{\text {a }}$ [35] that are used to constrain the photon mass. Sky position is given in right ascension, $\alpha$, and declination, $\delta$, at vernal equinox of $\mathrm{J} 2000.0$ epoch. Dispersion measure is in unit of $\mathrm{pc} \mathrm{cm}^{-3}$, where $\mathrm{DM}_{\mathrm{obs}}$ is from the fitting of the $v^{-2}$ behavior in the frequency-dependent time delay, and $\mathrm{DM}_{\mathrm{NE} 2001}$ is based on the NE2001 Galactic electron density model [36]. $z_{\max }$ is the upper limit on the true redshift, obtained by assuming that the excess dispersion measure, $\mathrm{DM}_{\mathrm{excess}} \equiv \mathrm{DM}_{\mathrm{obs}}-\mathrm{DM}_{\mathrm{NE2} 201}$, entirely comes from the IGM; since here we consistently use the parameter given in the main text and the full expression of $H_{e}(z)$, their values are close to, but larger than, that given in the catalog [35].

\begin{tabular}{|c|c|c|c|c|c|}
\hline FRB & Telescope & Sky position $(\alpha, \delta)_{\mathrm{J} 2000}$ & $\mathrm{DM}_{\mathrm{obs}}$ & $\mathrm{DM}_{\mathrm{NE} 2001}$ & $z_{\max }$ \\
\hline 010125 [37] & Parkes & $\left(19^{\mathrm{h}} 06^{\mathrm{m}} 53^{\mathrm{s}},-40^{\circ} 37^{\prime} 14^{\prime \prime}\right)$ & $790.3 \pm 0.3$ & 110 & 0.77 \\
\hline $010621[38]$ & Parkes & $\left(18^{\mathrm{h}} 52^{\mathrm{m}} 05^{\mathrm{s}},-08^{\circ} 29^{\prime} 35^{\prime \prime}\right)$ & $748 \pm 3$ & 523 & 0.27 \\
\hline 010724 [23] & Parkes & $\left(01^{\mathrm{h}} 18^{\mathrm{m}} 06^{\mathrm{s}},-75^{\circ} 12^{\prime} 18^{\prime \prime}\right)$ & $375 \pm 3$ & 44.58 & 0.38 \\
\hline 090625 [25] & Parkes & $\left(03^{\mathrm{h}} 07^{\mathrm{m}} 47^{\mathrm{s}},-29^{\circ} 55^{\prime} 36^{\prime \prime}\right)$ & $899.55 \pm 0.01$ & 31.69 & 0.98 \\
\hline $110220[24]$ & Parkes & $\left(22^{\mathrm{h}} 34^{\mathrm{m}} 38^{\mathrm{s}},-12^{\circ} 23^{\prime} 45^{\prime \prime}\right)$ & $944.38 \pm 0.05$ & 34.77 & 1.02 \\
\hline 110523 [39] & GBT & $\left(21^{\mathrm{h}} 45^{\mathrm{m}} 12^{\mathrm{s}},-00^{\circ} 09^{\prime} 37^{\prime \prime}\right)$ & $623.30 \pm 0.06$ & 43.52 & 0.66 \\
\hline $110626[24]$ & Parkes & $\left(21^{\mathrm{h}} 03^{\mathrm{m}} 43^{\mathrm{s}},-44^{\circ} 44^{\prime} 19^{\prime \prime}\right)$ & $723.0 \pm 0.3$ & 47.46 & 0.76 \\
\hline 110703 [24] & Parkes & $\left(23^{\mathrm{h}} 30^{\mathrm{m}} 51^{\mathrm{s}},-02^{\circ} 52^{\prime} 24^{\prime \prime}\right)$ & $1103.6 \pm 0.7$ & 32.33 & 1.20 \\
\hline 120127 [24] & Parkes & $\left(23^{\mathrm{h}} 15^{\mathrm{m}} 06^{\mathrm{s}},-18^{\circ} 25^{\prime} 38^{\prime \prime}\right)$ & $553.3 \pm 0.3$ & 31.82 & 0.60 \\
\hline 121002 [25] & Parkes & $\left(18^{\mathrm{h}} 14^{\mathrm{m}} 47^{\mathrm{s}},-85^{\circ} 11^{\prime} 53^{\prime \prime}\right)$ & $1629.18 \pm 0.02$ & 74.27 & 1.77 \\
\hline $121102[40]$ & Arecibo, GBT & $\left(05^{\mathrm{h}} 32^{\mathrm{m}} 09^{\mathrm{s}},+33^{\circ} 05^{\prime} 13^{\prime \prime}\right)$ & $557 \pm 2$ & 188 & 0.43 \\
\hline $130626[25]$ & Parkes & $\left(16^{\mathrm{h}} 27^{\mathrm{m}} 06^{\mathrm{s}},-07^{\circ} 27^{\prime} 48^{\prime \prime}\right)$ & $952.4 \pm 0.1$ & 66.87 & 1.00 \\
\hline $130628[25]$ & Parkes & $\left(09^{\mathrm{h}} 03^{\mathrm{m}} 02^{\mathrm{s}},+03^{\circ} 26^{\prime} 16^{\prime \prime}\right)$ & $469.88 \pm 0.01$ & 52.58 & 0.48 \\
\hline 130729 [25] & Parkes & $\left(13^{\mathrm{h}} 41^{\mathrm{m}} 21^{\mathrm{s}},-05^{\circ} 59^{\prime} 43^{\prime \prime}\right)$ & $861 \pm 2$ & 31 & 0.93 \\
\hline $131104[41]$ & Parkes & $\left(06^{\mathrm{h}} 44^{\mathrm{m}} 10^{\mathrm{s}},-51^{\circ} 16^{\prime} 40^{\prime \prime}\right)$ & $779 \pm 3$ & 71.1 & 0.80 \\
\hline 140514 [42] & Parkes & $\left(22^{\mathrm{h}} 34^{\mathrm{m}} 06^{\mathrm{s}},-12^{\circ} 18^{\prime} 46^{\prime \prime}\right)$ & $562.7 \pm 0.6$ & 34.9 & 0.60 \\
\hline 150418 [29] & Parkes & $\left(07^{\mathrm{h}} 16^{\mathrm{m}} 35^{\mathrm{s}},-19^{\circ} 00^{\prime} 40^{\prime \prime}\right)$ & $776.2 \pm 0.5$ & 188.5 & 0.67 \\
\hline $150807[43]$ & Parkes & $\left(22^{\mathrm{h}} 40^{\mathrm{m}} 23^{\mathrm{s}},-55^{\circ} 16^{\prime}\right)$ & $266.5 \pm 0.1$ & 70 & 0.23 \\
\hline 160317 [44] & UTMOST & $\left(07^{\mathrm{h}} 53^{\mathrm{m}} 47^{\mathrm{s}},-29^{\circ} 36^{\prime} 31^{\prime \prime}\right)$ & $1165 \pm 11$ & 319.6 & 0.95 \\
\hline 160410 [44] & UTMOST & $\left(08^{\mathrm{h}} 41^{\mathrm{m}} 25^{\mathrm{s}},+06^{\circ} 05^{\prime} 05^{\prime \prime}\right)$ & $278 \pm 3$ & 57.7 & 0.26 \\
\hline 160608 [44] & UTMOST & $\left(07^{\mathrm{h}} 36^{\mathrm{m}} 42^{\mathrm{s}},-40^{\circ} 47^{\prime} 52^{\prime \prime}\right)$ & $682 \pm 7$ & 238.3 & 0.51 \\
\hline
\end{tabular}

${ }^{a}$ http://www. astronomy. swin. edu.au/pulsar/frbcat/

the total dispersion measure reads,

$$
\mathrm{DM}_{\text {astro }}=\mathrm{DM}_{\mathrm{MW}}+\mathrm{DM}_{\mathrm{IGM}}+\mathrm{DM}_{\text {host }},
$$

where we have included contributions from the host galaxy and the near-source plasma (e.g. supernova remnant, pulsar wind nebula, HII region [48]) collectively in $\mathrm{DM}_{\text {host }}$. We will present in Section II B how different pieces in the above equation are treated in a Bayesian framework.

- With Eq. (2), it is straightforward to show that, in the $\Lambda \mathrm{CDM}$ universe, a photon with energy, $E=h v$, propagates slower relative to that with an infinite energy by [20-22, 49],

$$
\Delta t_{m_{\gamma}}=\frac{1}{2 H_{0}}\left(\frac{m_{\gamma} c_{0}^{2}}{h v}\right)^{2} H_{\gamma}(z)
$$

where $H_{0}=67.74 \pm 0.46 \mathrm{~km} \mathrm{~s}^{-1} \mathrm{Mpc}^{-1}$ is the Hubble constant [50], and $H_{\gamma}(z)$ is a dimensionless function of the source redshifit $z$ (see Figure 2),

$$
H_{\gamma}(z) \equiv \int_{0}^{z} \frac{1}{\left(1+z^{\prime}\right)^{2}} \frac{\mathrm{d} z^{\prime}}{\sqrt{\Omega_{\Lambda}+\left(1+z^{\prime}\right)^{3} \Omega_{\mathrm{m}}}},
$$

where the matter energy density $\Omega_{\mathrm{m}}=0.3089 \pm$ 0.0062 , and the vacuum energy density $\Omega_{\Lambda}=0.6911 \pm$ 0.0062 [50]. In deriving Eq. (5), we have assumed a flat universe with the curvature energy density $\Omega_{k}=0$, and the expansion of universe has been properly taken into account [20, 49].

In our hypothesis $\mathcal{H}$, the total time delay is,

$$
\Delta t=\Delta t_{\mathrm{DM}}+\Delta t_{\mathrm{m}_{\gamma}}
$$

The two terms in the above equation both depend on the frequency in the same way, therefore, it conforms to the observational fact that the total time delay $\Delta t \propto v^{-2}$. The observational dispersion measure, $\mathrm{DM}_{\mathrm{obs}}$, is obtained from the fit of the $v^{-2}$ behavior from the total time delay. In our hypothesis, it equals to,

$$
\mathrm{DM}_{\mathrm{obs}}=\mathrm{DM}_{\mathrm{astro}}+\mathrm{DM}_{\gamma}
$$


where $\mathrm{DM}_{\text {astro }}$ is given in Eq. (4), and we have denoted the "effective dispersion measure" caused by the non-vanishing photon mass as,

$$
\mathrm{DM}_{\gamma} \equiv \frac{4 \pi^{2} m_{e} \epsilon_{0} c_{0}^{5}}{h^{2} e^{2}} \frac{H_{\gamma}(z)}{H_{0}} m_{\gamma}^{2}
$$

\section{B. A Bayesian framework}

In Bayesian analysis, given data $\mathcal{D}$ and a hypothesis $\mathcal{H}$ (here Eq. 8), the posterior distribution of $m_{\gamma}^{2}$ can be obtained by,

$$
P\left(m_{\gamma}^{2} \mid \mathcal{D}, \mathcal{H}, \mathcal{I}\right)=\frac{P\left(\mathcal{D} \mid m_{\gamma}^{2}, \mathcal{H}, \mathcal{I}\right) P\left(m_{\gamma}^{2} \mid \mathcal{H}, \mathcal{I}\right)}{P(\mathcal{D} \mid \mathcal{H}, \mathcal{I})},
$$

where $\mathcal{I}$ is all other relevant prior background knowledge. In the above equation, given $\mathcal{H}$ and $\mathcal{I}, P\left(m_{\gamma}^{2} \mid \mathcal{H}, \mathcal{I}\right)$ is the prior on $m_{\gamma}^{2}, P\left(\mathcal{D} \mid m_{\gamma}^{2}, \mathcal{H}, \mathcal{I}\right) \equiv \mathcal{L}$ is the likelihood for the data, and $P(\mathcal{D} \mid \mathcal{H}, \mathcal{I})$ is the model evidence.

We choose a uniform prior on $m_{\gamma}$ in the range $m_{\gamma} \in$ $\left[10^{-69}, 10^{-42}\right] \mathrm{kg}$. The lower end is chosen because it corresponds to the ultimate upper limit that in principle we can probe in one observation due to the uncertainty principle of quantum mechanics and the finite age of our universe [51], while the upper end is chosen because beyond which the approximation in Eq. (2) breaks down. Such a wide prior across 27 orders of magnitude in $m_{\gamma}$ reflects our conservativeness.

To display the likelihood $\mathcal{L}$ that we adopt in our calculation, we first investigate different contributions in Eq. (4) -

- $\mathrm{DM}_{\mathrm{MW}}$ : albeit with uncertainties, there are electron distribution models for the Milky Way that incorporate different astrophysical observational results [36, 52]. We here use the NE2001 model. For different FRBs, $\mathrm{DM}_{\mathrm{MW}}$ is calculated from their line of sight. We assign a $20 \%$ uncertainty to the value given by the NE2001 model to account for possible model inaccuracy.

In principle, there is an additional contribution from the Galactic halo, which is not captured by the NE2001 model because pulsars in general do not probe this regime [36]. The halo contribution is not easy to model, but in our case it could already have been included $e f$ fectively in $\mathrm{DM}_{\text {host }}$ [48], which is obtained from the excess dispersion measure of FRBs, $\mathrm{DM}_{\text {excess }} \equiv \mathrm{DM}_{\mathrm{obs}}-$ $\mathrm{DM}_{\mathrm{NE} 2001}$ (see below). The large uncertainty that we assign to $\mathrm{DM}_{\mathrm{MW}}$ could also account for (at least part of) this unknown contribution.

- $\mathrm{DM}_{\mathrm{IGM}}$ : the dispersion measure due to the intergalactic medium (IGM) is given by [47],

$\mathrm{DM}_{\mathrm{IGM}}=\frac{3 c_{0} H_{0} \Omega_{\mathrm{b}} f_{\mathrm{IGM}}}{8 \pi G m_{\mathrm{p}}} \int_{0}^{z} \frac{g(z)\left(1+z^{\prime}\right) \mathrm{d} z^{\prime}}{\sqrt{\Omega_{\Lambda}+\left(1+z^{\prime}\right)^{3} \Omega_{\mathrm{m}}}}$

where $m_{\mathrm{p}}$ is the mass of a proton, $\Omega_{\mathrm{b}}=0.0486 \pm 0.0007$ is the baryonic matter energy density [50], $f_{\mathrm{IGM}} \simeq 0.83$ is its fraction to the IGM [53], and

$$
g(z) \equiv \frac{3}{4} y_{1} \chi_{e, \mathrm{H}}(z)+\frac{1}{8} y_{2} \chi_{e, \mathrm{He}}(z),
$$

where $y_{1} \sim 1, y_{2} \sim 1$, and the ionized fractions of IGM $\chi_{e, \mathrm{H}}(z) \simeq 1$ for hydrogen at $z<6[54]$ and $\chi_{e, \mathrm{He}}(z) \simeq 1$ for helium at $z<2$ [55]. Therefore for $z<2$, we have,

$$
\mathrm{DM}_{\mathrm{IGM}} \simeq \frac{3 c_{0} H_{0} \Omega_{e, \mathrm{IGM}}}{8 \pi G m_{\mathrm{p}}} H_{e}(z)
$$

where the effective energy density of ionized baryons $\Omega_{e, \mathrm{IGM}} \simeq 0.035[47,56,57]$. To be conservative, we associate a $20 \%$ uncertainty to $\Omega_{e, \text { IGM }}[21,48]$, in the hope that such a large uncertainty could account for, at least partially, the inhomogeneity of IGM along different line of sight. In Eq. (13), the dimensionless redshift function, $H_{e}(z)$, reads,

$$
H_{e}(z) \equiv \int_{0}^{z} \frac{\left(1+z^{\prime}\right) \mathrm{d} z^{\prime}}{\sqrt{\Omega_{\Lambda}+\left(1+z^{\prime}\right)^{3} \Omega_{\mathrm{m}}}}
$$

In Figure 2 we depict $H_{e}(z)$ and $H_{\gamma}(z)$ with cosmological parameters from the $\Lambda$ CDM model [50]. Worthy to mention that, Bonetti et al. [21, 22] pointed out that the different behavior of these two redshift functions might be able to break parameter degeneracy in testing the photon mass at the point when a handful measurements of redshift for FRBs become available. For now we leave this point to future work.

To predict the contribution of $\mathrm{DM}_{\mathrm{IGM}}$, we need a redshift measurement, which is only available for FRB 121102 up to now [32]. ${ }^{2}$ Due to our lack of knowledge, we assume a prior for $z$ such that the prior for the FRB's spatial distribution is uniform in the comoving spherical volume,

$$
\left.V_{\mathrm{c}}^{\max } \equiv \frac{4 \pi}{3} D_{\mathrm{c}}^{3}(z)\right|_{z=z_{\max }},
$$

where $D_{c}(z)$ is the comoving distance to a source at redshift $z$,

$$
D_{\mathrm{c}}(z) \equiv \frac{c_{0}}{H_{0}} \int_{0}^{z} \frac{\mathrm{d} z^{\prime}}{\sqrt{\Omega_{\Lambda}+\left(1+z^{\prime}\right)^{3} \Omega_{\mathrm{m}}}},
$$

and $z_{\max }$ is the maximum possible redshift value of redshift (see Table I) by setting $\mathrm{DM}_{\text {host }}=0$ and $m_{\gamma}=0$ in Eq. (8) [35]. Such a prior for $z$ will be denoted as uniform for $V_{\mathrm{c}}(z) \in\left[0, V_{\mathrm{c}}^{\max }\right]$ where $V_{\mathrm{c}}(z) \equiv \frac{4 \pi}{3} D_{\mathrm{c}}^{3}(z)$ is the comoving spherical volume within redshift $z$. In

\footnotetext{
${ }^{2}$ We will not consider the redshift measurement for FRB 150418 [29], which is challenged with a flare in an active galactic nucleus [30-32]. Nevertheless, the inclusion of this measurement into our framework is straightforward if the redshift measurement is proven genuine.
} 
Section IV we will in addition present the results with a prior of $z$ that traces the star formation rate [58], and confirm the robustness of results. For FRB 121102, the measured redshift will be used as the prior for $z$ (see Section III A).

- $\mathrm{DM}_{\text {host }}$ : From a $\mathrm{DM}_{\text {excess }}-F_{v}$ relation, Yang et al. [48] derived a statistical result, $\left\langle\mathrm{DM}_{\text {host }}\right\rangle=267_{-111}^{+173} \mathrm{pc} \mathrm{cm}^{-3}$ in the rest frame of FRB, under the assumption that the isotropic-equivalent luminosity of FRBs has a characteristic value. Simulations show that a 30\% Gaussian dispersion in $\mathrm{DM}_{\text {host }}$ still keeps the result valid [48]. The conclusion in Yang et al. [48] is supported by the large scattering time of FRBs and the inferred $\mathrm{DM}_{\text {host }}$ from FRB 121102 [32, 48]. We will use this result in our estimation of $\mathrm{DM}_{\text {host }}$, after multiplying it by a factor, $(1+z)^{-1}$, which takes the cosmological evolution into account. ${ }^{3}$

Finally the logarithm of likelihood is constructed as,

$$
\ln \mathcal{L}=-\frac{1}{2} \sum_{i} \frac{\left(\mathrm{DM}_{\mathrm{obs}}^{i}-\mathrm{DM}_{\text {astro }}^{i}-\mathrm{DM}_{\gamma}^{i}\right)^{2}}{\sigma_{i}^{2}},
$$

where $i$ indexes FRBs, $\mathrm{DM}_{\text {astro }}$ is the dispersion measure obtained with the above listed prescriptions in Markov-chain Monte Carlo (MCMC) runs, $\sigma$ includes all uncertainties added in quadratic (including uncertainties in $\mathrm{DM}_{\mathrm{obs}}, \mathrm{DM}_{\mathrm{MW}}$, $\mathrm{DM}_{\mathrm{IGM}}$, and $\mathrm{DM}_{\text {host }}$ ), and $\mathrm{DM}_{\gamma}$ denotes the second term in Eq. (8). In writing Eq. (17), an assumption is made that the observations of different FRBs are independent.

\section{RESULTS}

As said, we use MCMC techniques to explore the posterior in Eq. (10). Ideally, one would use the log-likelihood in Eq. (17) to simultaneously analyze all FRBs in one go, whereas here the computational cost would be very high due to the large dimensionality of the parameter space. The dimensionality equals to the number of FRBs (their redshifts) plus one (the photon mass $m_{\gamma}$ ). We adopt a sub-optimal strategy where the posteriors of $m_{\gamma}$, from different individual FRBs, are combined after independent MCMC run is performed on each single FRB [59]. This is not a down-graded choice because we know that the redshifts of different FRBs

\footnotetext{
${ }^{3}$ Strictly speaking, the $\mathrm{DM}_{\text {host }}$ obtained in Yang et al. [48] uses the assumption $m_{\gamma}=0$. A global re-analysis that closely follows the MCMC simulations in that work but allowing a nonzero $m_{\gamma}$ would be ideal, however, this goes beyond the scope of current work. Instead we perform the following simulation to assess the influence to our result from using the $\mathrm{DM}_{\text {host }}$ in Yang et al. [48]. Notice that, when $\mathrm{DM}_{\text {host }}$ is underestimated, the constraint on $m_{\gamma}$ is more conservative. Therefore, we perform the most conservative simulation that artificially sets $\mathrm{DM}_{\text {host }}=0$. We observe that, even under such an assumption, our results only change by a factor less than three. Consequently, the results in the paper are robust to possible changes in the $\mathrm{DM}_{\text {host }}$ value we adopt.
}

are unlikely to correlate with each other. Such an approach is also the strategy adopted in constraining the strong equivalence principle in Ref. [60], the local Lorentz invariance of gravity in Refs. [61, 62], and the parameterized tests of general relativity with the Advanced LIGO events in Ref. [63].

We use the PYTHON implementation of an affine-invariant MCMC ensemble sampler [64, 65], EMCEE, ${ }^{4}$ to explore the posterior distributions. This algorithm generally has better performance over the traditional MCMC sampling methods (e.g., the Metropolis-Hasting algorithm), as measured by the smaller autocorrelation time and fewer hand-tuning parameters [65]. We set up MCMC runs to investigate the $\left(m_{\gamma}, z\right)$ pair for each FRB. As mentioned, the priors are uniform in $V_{\mathrm{c}}(z) \in\left[0, V_{\mathrm{c}}^{\max }\right]$ and in $m_{\gamma} \in\left[10^{-69}, 10^{-42}\right] \mathrm{kg}$. Each MCMC run samples the posterior distribution according to Eq. (10), with the log-likelihood given by Eq. (17), with 20 chains. For each FRB, $10^{6}$ samples are accumulated. The first half of the samples are discarded as the BURN-IN phase [66]. We check the convergence of different chains with the Gelman-Rubin statistic [67],

$$
\hat{R} \equiv \sqrt{\frac{\widehat{\operatorname{Var}(\Theta \mid \mathcal{D})}}{W},}
$$

where the estimate of the marginal posterior variance for each parameter $\Theta \equiv\left\{\theta_{i j}\right\}$ (indices $i, j$ denote the $i$-th posterior sample in the $j$-th chain) is,

$$
\widehat{\operatorname{Var}}(\Theta \mid \mathcal{D}) \equiv \frac{N-1}{N} W+\frac{1}{N} B,
$$

with the between-chain variance, $B$, and the within-chain variance, $W$,

$$
\begin{aligned}
B & \equiv \frac{N}{M-1} \sum_{j=1}^{M}\left(\frac{1}{N} \sum_{i=1}^{N} \theta_{i j}-\frac{1}{N} \sum_{i=1}^{N} \frac{1}{M} \sum_{k=1}^{M} \theta_{i k}\right)^{2}, \\
W & \equiv \frac{1}{M} \sum_{j=1}^{M}\left[\frac{1}{N-1} \sum_{i=1}^{N}\left(\theta_{i j}-\frac{1}{N} \sum_{k=1}^{N} \theta_{k j}\right)^{2}\right],
\end{aligned}
$$

where $M$ and $N$ are respectively the number of chains (in our case $M=20$ ) and the number of samples per chain (in our case $N=25000$ after discarding the BURN-IN samples). Our convergence test shows $\hat{R} \lesssim 1.002$ for all cases, indicating very good convergence in MCMC runs. The posteriors and constraints on $m_{\gamma}$ are presented in the following subsections.

\section{A. Limit from FRB 121102}

Because the redshift of FRB 121102 was measured to great precision in Refs. [32, 33], $z=0.19273 \pm 0.00008$, we would like to compare the constraints on the photon mass with and

\footnotetext{
${ }^{4}$ http://dan.iel.fm/emcee
} 

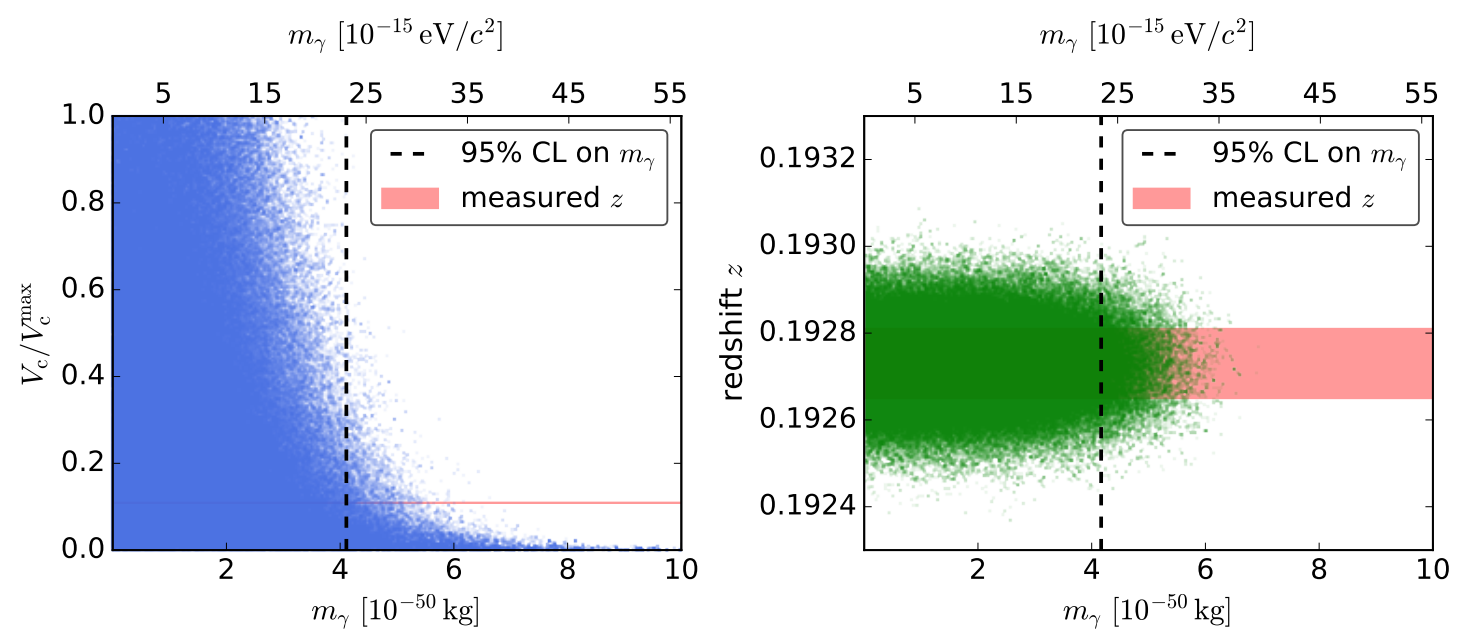

FIG. 3. Distribution of MCMC samples from FRB 121102 (left) in the $m_{\gamma}-\left(V_{\mathrm{c}} / V_{\mathrm{c}}^{\max }\right)$ plane: without using the redshift measurement, and (right) in the $m_{\gamma}-z$ plane using the redshift measurement as the prior on $z$ [32]. The pink bands show the redshift measurement and its 1- $\sigma$ uncertainty obtained by Chatterjee et al. [32]; the band of $V_{\mathrm{c}}(z) / V_{\mathrm{c}}^{\max }$ is too narrow to be visible in the left panel. The 95\% CL constraints on $m_{\gamma}$ are depicted as dashed lines.

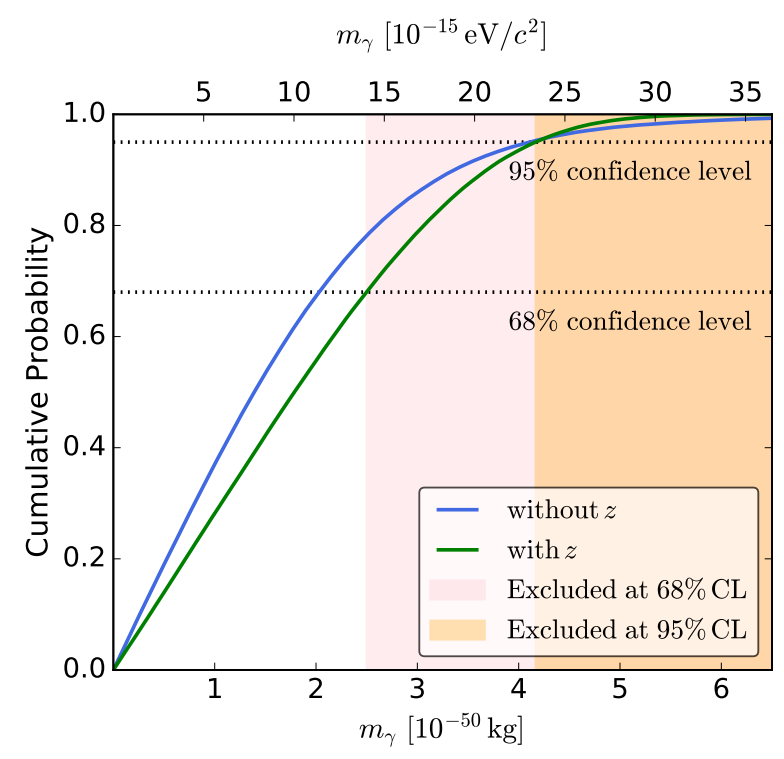

FIG. 4. The cumulative posterior probability distributions on $m_{\gamma}$ from FRB 121102 without using the redshift measurement (in blue) and using the redshift measurement (in green). The excluded values for $m_{\gamma}$ at $68 \%$ and $95 \%$ CLs are shown with shadowed areas for the case where the redshift is used.

without this measurement. By including the redshift measurement, we mean using a Gaussian prior for $z$, centered around its measured value with a spread of the uncertainty.

In Figure 3 we show the samples returned by the MCMC sampler (after discarding the BURN-IN samples) in both cases for FRB 121102. We immediately see that if the samples are marginalized over the photon mass, priors on $z$ are more or less recovered in both cases. This means that the Bayesian framework proposed here does not add more information to the redshift, as it should not.

In Figure 4 we show the accumulative posterior probability on $m_{\gamma}$, marginalized over the redshift $z$, for both cases. As we can see, the result from the use of redshift measurement is very close to the one that does not use the redshift. We read out, at a $95 \%$ confidence level,

$$
m_{\gamma} \leq 4.1 \times 10^{-50} \mathrm{~kg},
$$

when using an uninformative uniform prior on $V_{\mathrm{c}}(z)$, and

$$
m_{\gamma} \leq 4.2 \times 10^{-50} \mathrm{~kg},
$$

when $z=0.19273 \pm 0.00008$ is used. The latter agrees well with the result presented by Bonetti et al. [22] for this FRB with a less sophisticated method. The marginalized $1 \mathrm{D}$ probability distribution on $m_{\gamma}$ with the uninformative prior has a long tail which reflects our ignorance in the redshift. The ultimate closeness of the results in Eqs. (22-23) is a bit coincident, but it also shows the reasonableness of the use of the uninformative prior.

\section{B. Limits from individual FRBs}

Except for FRB 121102 discussed above, the other 20 FRBs in the catalog (see Table I) unfortunately have no redshift measurement [35]. Therefore, we can only rely on the uninformative priors. The distribution of MCMC samples are shown in Figure 5 for these FRBs. As in the FRB 121102 case with the uninformative prior, the distributions have long tails towards large $m_{\gamma}$. Especially for the FRBs with large $z_{\max }$ (e.g., FRB 110703 and FRB 121002), a large $m_{\gamma}$ is needed to account for part of the dispersion measure in $\mathrm{DM}_{\mathrm{obs}}-\mathrm{DM}_{\mathrm{MW}}$ 

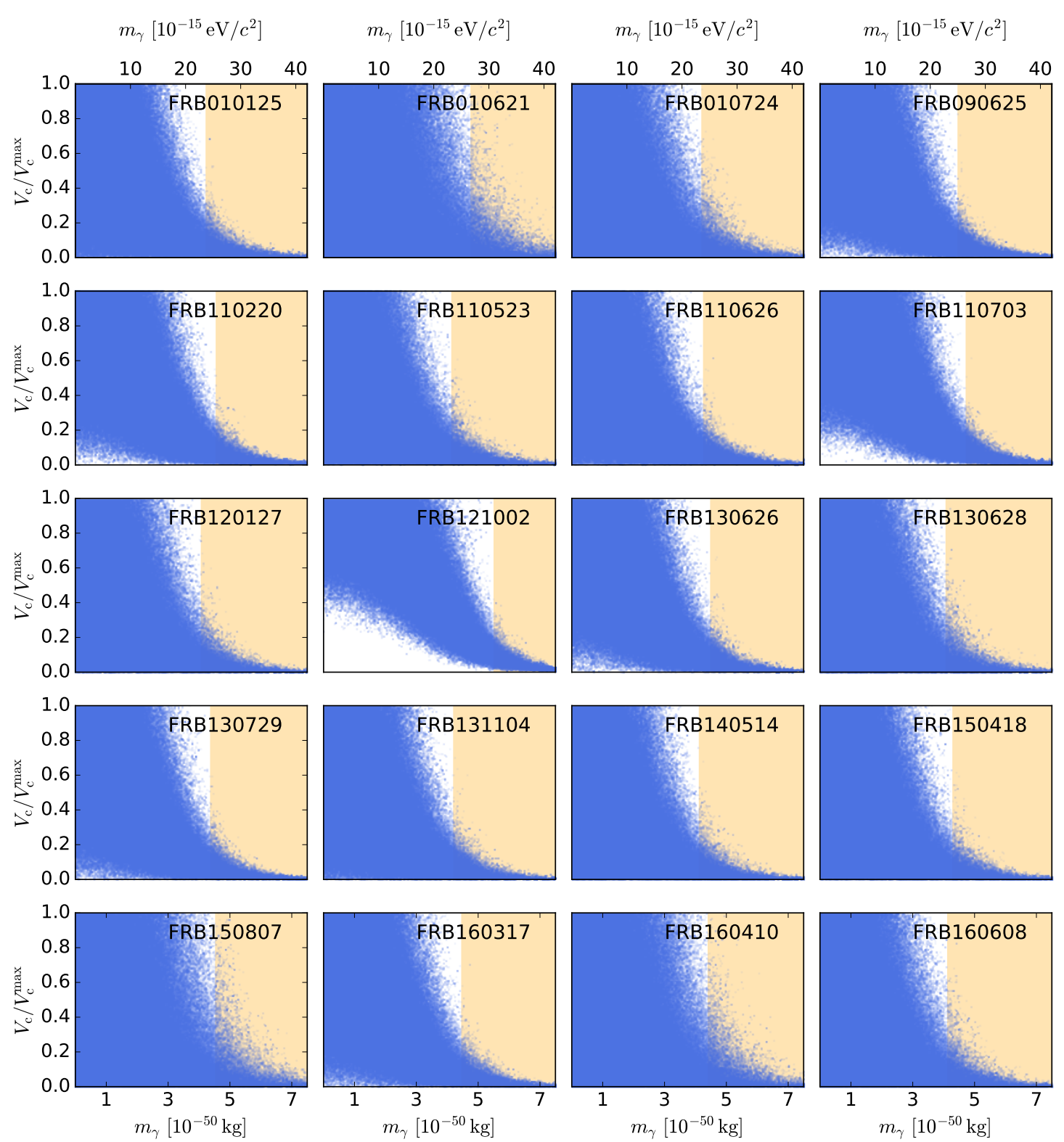

FIG. 5. The MCMC samples in the $m_{\gamma}-\left(V_{\mathrm{c}} / V_{\mathrm{c}}^{\max }\right)$ plane for a catalog of FRBs in Table I except FRB 121102. The excluded regions at 95\% CL are shadowed for each FRB.

when the redshift is very small, as expected. From their panels in Figure 5, we see that some regions with small $z$ and small $m_{\gamma}$ have no support from MCMC runs. Because of the conservatively large uncertainties that we use in $\mathrm{DM}_{\mathrm{MW}}$ and in $\mathrm{DM}_{\mathrm{IGM}}$, these FRBs individually only constrain $m_{\gamma}$ at $\sim 5 \times 10^{-50} \mathrm{~kg}$ at $95 \%$ confidence level, as shown by the shadowed regions in the figure. The uncertainties in these two terms dominate the test, hence, in terms of order of magnitude, all FRBs here have comparable constraints.

\section{Combined limit from a catalog of FRBs}

We now have 21 individual constraints on $m_{\gamma}$. Assuming that these FRBs are independent, we can combine their posterior distributions, in the spirit of Eq. (10). Similar combination of posteriors was done in Refs. [60-63] under different subjects. Here, since for FRB 121102 a reliable redshift is available $[32,33]$, we use the result that takes this measurement into account. In Figure 6, we plot the marginalized accumulative posterior distributions for 20 FRBs (see Figure 5) in gray, that generally give $m_{\gamma} \lesssim 5 \times 10^{-50} \mathrm{~kg}$ at $95 \%$ confidence level, and the accumulative posterior distribution for FRB 121102 in green (same as the green curve in Figure 4), 


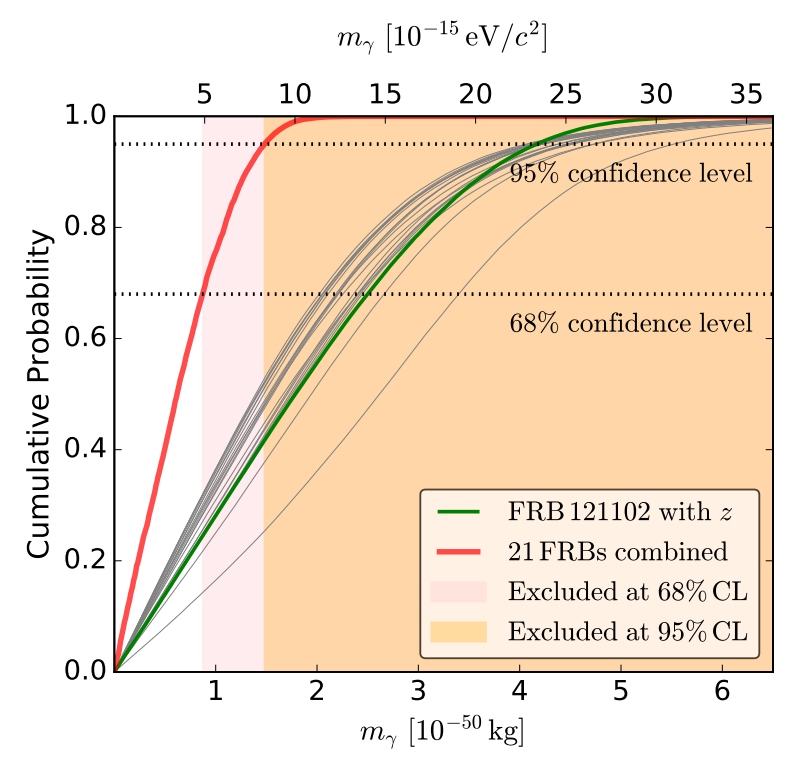

FIG. 6. The cumulative posterior probability distributions for 20 FRBs (the catalog of FRBs in Table I except FRB 121102) are shown in grey. The same quantity is shown in green for FRB 121102 (the same green curve in Figure 4). The combination of these 21 FRBs is given in red. The excluded values for $m_{\gamma}$ at $68 \%$ and $95 \%$ CLs are shown with shadowed areas for the combination.

that gives $m_{\gamma} \leq 4.2 \times 10^{-50} \mathrm{~kg}$ at $95 \%$ confidence level (see Eq. 23). We also give the accumulative posterior distribution that combines the 21 FRBs with a red curve in the figure. In the Bayesian sense, it is unlikely that multiple FRBs reside in the long tails of their distributions. This result demonstrates the collective power of these "deceptively boring" FRBs that have no redshift measurement. It has strong implication for future study using FRBs to constrain the photon mass. The final combination of 21 FRBs (the red curve in Figure 6) give a tight constraint on $m_{\gamma}$,

$$
\begin{aligned}
m_{\gamma} & \leq 8.7 \times 10^{-51} \mathrm{~kg} \simeq 4.9 \times 10^{-15} \mathrm{eV} / \mathrm{c}^{2}, \\
m_{\gamma} & \leq 1.5 \times 10^{-50} \mathrm{~kg} \simeq 8.4 \times 10^{-15} \mathrm{eV} / \mathrm{c}^{2},
\end{aligned}
$$

at $68 \%$ and $95 \%$ confidence levels respectively. These limits improve over previous results that only used a single FRB [20-22] by a factor of $\sim 4$.

\section{DISCUSSIONS}

Recently, the first direct observation of gravitational waves from a binary black hole merger at a cosmological distance by the Advanced LIGO puts a constraint on the graviton mass, $m_{g} \leq 1.2 \times 10^{-22} \mathrm{eV} / c^{2}$, at $90 \%$ confidence level [68]. Because most of the power of the gravitational-wave event is at $O\left(10^{2}\right) \mathrm{Hz}$, even significantly lower than the $O\left(10^{9}\right) \mathrm{Hz}$ radio waves we here use to constrain the photon mass, a tighter constraint on the mass is expected (cf. discussion below Eq. 2). Nevertheless, the constraints on the photon mass (see Eqs.
24-25) pertain to a different sector of species. As far as we are aware, this is the tightest limit on the photon mass that is obtained solely depending on the propagation kinematics, therefore completely avoiding assumptions about the underlying dynamical theory for the massive photon.

The Bayesian framework proposed here will be even more valuable in future, when more and more FRB observations become available (for example, with the ALERT project ${ }^{5}$ ). The improvement could come from -

1. new discovery of more FRBs;

2. more coincident measurements of FRB redshift;

3. a better understanding of the various astrophysical contributions to the observed dispersion measure, including those from the Milky Way, the IGM, and the host galaxies of FRBs.

If future FRBs are observed with the same quality as current ones, we expect a rough $\mathcal{N}^{-1 / 2}$ improvement on the photon mass where $\mathcal{N}$ is the number of FRBs. The improvement from the measurement of the redshift is very hard to predict. It depends on the redshift value that is measured. For example, in the case of FRB 121102, the measured redshift $z \simeq 0.19$ resides in the lower end of its possible values up to $z_{\max } \simeq 0.43$. Taking the uniform prior in volume into account, one would expect a chance of approximately $0.19^{3} / 0.43^{3} \simeq 9 \%$ to observe a redshift as low as 0.19 for FRB 121102. ${ }^{6}$ Even in such a case, the inclusion of the measured redshift does not provide a worse constraint compared with the case where we are uninformative about the redshift. Were the measured redshift larger, the constraint would be better. For now, the constraint on the photon mass is limited by our assumptions about the uncertainties from the Milky Way, the IGM, and the host galaxies of FRBs (cf. Section II B). Better determination of these contributions leads to tighter limits for individual FRBs, and when combined through Eq. (10), a better combined constraint results. We expect all three points listed above will make progresses in observations soon.

The reason that in our analysis those FRBs without the redshift measurement still contribute to the constraint is the use of an uninformative prior for the redshift and the inclusion of it in a Bayesian way. In addition to the uniform-in-volume prior used in Section III, we here use another physical prior that traces the star formation rate for a robustness test. We use the fit for the star formation rate given by Yüksel et al. [58],

$$
\dot{\rho}_{*}(z)=\dot{\rho}_{0}\left[(1+z)^{a \eta}+\left(\frac{1+z}{B}\right)^{b \eta}+\left(\frac{1+z}{C}\right)^{c \eta}\right]^{1 / \eta},
$$

where $\dot{\rho}_{0}=0.02 M_{\odot} \mathrm{yr}^{-1} \mathrm{Mpc}^{-3}, a=3.4, b=-0.3, c=-3.5$, $\eta=-10, B=\left(1+z_{1}\right)^{1-a / b}, C=\left(1+z_{1}\right)^{(b-a) / c}\left(1+z_{2}\right)^{1-b / c}$ with the breaking points $z_{1}=1$ and $z_{2}=4$. We obtain a

\footnotetext{
5 http: //www . alert. eu.org/

${ }^{6}$ For sources with low redshift, the comoving distance $\propto z$, thus the comoving volume $\propto z^{3}$.
} 
combined limit $7.5 \times 10^{-51} \mathrm{~kg}\left(1.3 \times 10^{-50} \mathrm{~kg}\right)$ at $68 \%(95 \%)$ confidence level, showing that reasonable changes in the prior of redshift do not lead to large difference. The slight improvement here results from the fact that the star formation rate in Eq. (26) favours larger $z$ when $z \leq 1$ where most FRBs in Table I reside.
Lastly, worthy to mention that, because FRBs are distributed nearly isotropically in the sky (see Figure 1), they will also be useful to constrain the anomalous anisotropic inertial mass tensor of photons [69-71] in a similar way that pulsars are used to set constraints on a Lorentz-violating tensor [72]. We leave this point for future work.
[1] A. Einstein, Annalen Phys. 17, 891 (1905).

[2] S. Weinberg, The Quantum theory of fields. Vol. 1: Foundations (Cambridge University Press, 2005).

[3] A. Proca, J. Phys. Radium 7, 347 (1936).

[4] L. de Broglie, J. Phys. Radium 3, 422 (1922).

[5] H. Georgi, P. H. Ginsparg, and S. L. Glashow, Nature 306, 765 (1983).

[6] A. S. Goldhaber and M. M. Nieto, Rev. Mod. Phys. 82, 939 (2010), arXiv:0809.1003 [hep-ph].

[7] G. Spavieri, J. Quintero, G. T. Gillies, and M. Rodriguez, Eur. Phys. J. D61, 531 (2011).

[8] E. R. Williams, J. E. Faller, and H. A. Hill, Phys. Rev. Lett. 26, 721 (1971).

[9] N. M. Kroll, Phys. Rev. Lett. 27, 340 (1971).

[10] D. D. Lowenthal, Phys. Rev. D8, 2349 (1973).

[11] G. V. Chibisov, Sov. Phys. Usp. 19, 624 (1976), [Usp. Fiz. Nauk 119, 551 (1976)].

[12] L. Davis, Jr., A. S. Goldhaber, and M. M. Nieto, Phys. Rev. Lett. 35, 1402 (1975).

[13] R. Lakes, Phys. Rev. Lett. 80, 1826 (1998).

[14] J. Luo, L.-C. Tu, Z.-K. Hu, and E.-J. Luan, Phys. Rev. Lett. 90, 081801 (2003).

[15] D. D. Ryutov, Plasma Phys. Control. Fusion 39, A73 (1997).

[16] D. D. Ryutov, Plasma Phys. Control. Fusion 49, B429 (2007).

[17] P. Pani, V. Cardoso, L. Gualtieri, E. Berti, and A. Ishibashi, Phys. Rev. Lett. 109, 131102 (2012), arXiv:1209.0465 [gr-qc].

[18] Y.-P. Yang and B. Zhang, (2017), arXiv:1701.03034 [astroph.HE].

[19] B. Lovell, F. L. Whipple, and L. H. Solomon, Nature (London) 202, 377 (1964).

[20] X.-F. Wu, S.-B. Zhang, H. Gao, J.-J. Wei, Y.-C. Zou, W.-H. Lei, B. Zhang, Z.-G. Dai, and P. Mszros, Astrophys. J. 822, L15 (2016), arXiv:1602.07835 [astro-ph.HE].

[21] L. Bonetti, J. Ellis, N. E. Mavromatos, A. S. Sakharov, E. K. G. Sarkisyan-Grinbaum, and A. D. A. M. Spallicci, Phys. Lett. B757, 548 (2016), arXiv:1602.09135 [astro-ph.HE].

[22] L. Bonetti, J. Ellis, N. E. Mavromatos, A. S. Sakharov, E. K. Sarkisyan-Grinbaum, and A. D. A. M. Spallicci, Phys. Lett. B768, 326 (2017), arXiv:1701.03097 [astro-ph.HE].

[23] D. R. Lorimer, M. Bailes, M. A. McLaughlin, D. J. Narkevic, and F. Crawford, Science 318, 777 (2007), arXiv:0709.4301 [astro-ph].

[24] D. Thornton et al., Science 341, 53 (2013), arXiv:1307.1628 [astro-ph.HE].

[25] D. J. Champion et al., Mon. Not. Roy. Astron. Soc. 460, L30 (2016), arXiv:1511.07746 [astro-ph.HE].

[26] G. Amelino-Camelia, J. R. Ellis, N. E. Mavromatos, D. V. Nanopoulos, and S. Sarkar, Nature 393, 763 (1998), arXiv:astro-ph/9712103 [astro-ph].

[27] D. Mattingly, Living Rev. Rel. 8, 5 (2005), arXiv:grqc/0502097 [gr-qc].

[28] L. Shao, Z. Xiao, and B.-Q. Ma, Astropart. Phys. 33, 312 (2010), arXiv:0911.2276 [hep-ph].
[29] E. F. Keane et al., Nature 530, 453 (2016), arXiv:1602.07477 [astro-ph.HE].

[30] P. K. G. Williams and E. Berger, Astrophys. J. 821, L22 (2016), arXiv:1602.08434 [astro-ph.CO].

[31] H. K. Vedantham, V. Ravi, K. Mooley, D. Frail, G. Hallinan, and S. R. Kulkarni, Astrophys. J. 824, L9 (2016), arXiv:1603.04421 [astro-ph.HE].

[32] S. Chatterjee et al., Nature 541, 58 (2017), arXiv:1701.01098 [astro-ph.HE].

[33] S. P. Tendulkar et al., Astrophys. J. 834, L7 (2017), arXiv:1701.01100 [astro-ph.HE].

[34] D. Palaniswamy and B. Zhang, (2017), arXiv:1703.09232 [astro-ph.HE].

[35] E. Petroff, E. D. Barr, A. Jameson, E. F. Keane, M. Bailes, M. Kramer, V. Morello, D. Tabbara, and W. van Straten, Publ. Astron. Soc. Aust. 33, e045 (2016), arXiv:1601.03547 [astroph.HE].

[36] J. M. Cordes and T. J. W. Lazio, (2002), arXiv:astroph/0207156 [astro-ph].

[37] S. Burke-Spolaor and K. W. Bannister, Astrophys. J. 792, 19 (2014), arXiv:1407.0400 [astro-ph.HE].

[38] E. F. Keane, M. Kramer, A. G. Lyne, B. W. Stappers, and M. A. McLaughlin, Mon. Not. Roy. Astron. Soc. 415, 3065 (2011), arXiv:1104.2727 [astro-ph.SR].

[39] K. Masui et al., Nature 528, 523 (2015), arXiv:1512.00529 [astro-ph.HE].

[40] L. G. Spitler et al., Astrophys. J. 790, 101 (2014), arXiv:1404.2934 [astro-ph.HE].

[41] V. Ravi, R. M. Shannon, and A. Jameson, Astrophys. J. 799, L5 (2015), arXiv:1412.1599 [astro-ph.HE].

[42] E. Petroff et al., Mon. Not. Roy. Astron. Soc. 447, 246 (2015), arXiv:1412.0342 [astro-ph.HE].

[43] V. Ravi, R. M. Shannon, M. Bailes, K. Bannister, S. Bhandari, N. D. R. Bhat, S. Burke-Spolaor, M. Caleb, C. Flynn, A. Jameson, S. Johnston, E. F. Keane, M. Kerr, C. Tiburzi, A. V. Tuntsov, and H. K. Vedantham, Science 354, 1249 (2016), arXiv:1611.05758 [astro-ph.HE].

[44] M. Caleb et al., (2017), arXiv:1703.10173 [astro-ph.HE].

[45] J. I. Katz, Mod. Phys. Lett. A31, 1630013 (2016), arXiv:1604.01799 [astro-ph.HE].

[46] D. R. Lorimer and M. Kramer, Handbook of Pulsar Astronomy (Cambridge University Press, 2012).

[47] W. Deng and B. Zhang, Astrophys. J. 783, L35 (2014), arXiv:1401.0059 [astro-ph.HE].

[48] Y.-P. Yang, R. Luo, Z. Li, and B. Zhang, Astrophys. J. 839, L25 (2017), arXiv:1701.06465 [astro-ph.HE].

[49] U. Jacob and T. Piran, JCAP 0801, 031 (2008), arXiv:0712.2170 [astro-ph].

[50] P. A. R. Ade et al. (Planck), Astron. Astrophys. 594, A13 (2016), arXiv:1502.01589 [astro-ph.CO].

[51] J. D. Barrow and R. R. Burman, Nature 307, 14 (1984).

[52] J. M. Yao, R. N. Manchester, and N. Wang, Astrophys. J. 835, 29 (2017), arXiv:1610.09448. 
[53] M. Fukugita, C. J. Hogan, and P. J. E. Peebles, Astrophys. J. 503, 518 (1998), arXiv:astro-ph/9712020 [astro-ph].

[54] X.-H. Fan, C. L. Carilli, and B. G. Keating, Ann. Rev. Astron. Astrophys. 44, 415 (2006), arXiv:astro-ph/0602375 [astro-ph].

[55] M. McQuinn, A. Lidz, M. Zaldarriaga, L. Hernquist, P. F. Hopkins, S. Dutta, and C.-A. Faucher-Giguère, Astrophys. J. 694, 842 (2009), arXiv:0807.2799.

[56] K. Ioka, Astrophys. J. 598, L79 (2003), arXiv:astro-ph/0309200 [astro-ph].

[57] S. Inoue, Mon. Not. Roy. Astron. Soc. 348, 999 (2004), arXiv:astro-ph/0309364 [astro-ph].

[58] H. Yüksel, M. D. Kistler, J. F. Beacom, and A. M. Hopkins, Astrophys. J. 683, L5 (2008), arXiv:0804.4008 [astro-ph].

[59] L. Lyons and E. Chapon, (2017), arXiv:1704.05540 [physics.data-an].

[60] I. H. Stairs et al., Astrophys. J. 632, 1060 (2005), arXiv:astro$\mathrm{ph} / 0506188$ [astro-ph].

[61] L. Shao, R. N. Caballero, M. Kramer, N. Wex, D. J. Champion, and A. Jessner, Class. Quant. Grav. 30, 165019 (2013), arXiv:1307.2552 [gr-qc].

[62] L. Shao, Phys. Rev. D90, 122009 (2014), arXiv:1412.2320 [gr- qc].

[63] B. P. Abbott et al. (Virgo, LIGO Scientific), Phys. Rev. X6, 041015 (2016), arXiv:1606.04856 [gr-qc].

[64] J. Goodman and J. Weare, Comm. App. Math. and Comp. Sci. 5, 65 (2010).

[65] D. Foreman-Mackey, D. W. Hogg, D. Lang, and J. Goodman, Publ. Astron. Soc. Pac. 125, 306 (2013), arXiv:1202.3665 [astro-ph.IM].

[66] S. Brooks, A. Gelman, G. Jones, and X. Meng, Handbook of Markov Chain Monte Carlo (CRC Press, 2011).

[67] A. Gelman and D. B. Rubin, Statist. Sci. 7, 457 (1992).

[68] B. P. Abbott et al. (LIGO Scientific, Virgo), Phys. Rev. Lett. 116, 221101 (2016), arXiv:1602.03841 [gr-qc].

[69] C. Laemmerzahl, Class. Quant. Grav. 15, 13 (1998).

[70] V. A. Kostelecky and M. Mewes, Phys. Rev. D66, 056005 (2002), arXiv:hep-ph/0205211 [hep-ph].

[71] V. A. Kostelecky and M. Mewes, Phys. Rev. D80, 015020 (2009), arXiv:0905.0031 [hep-ph].

[72] L. Shao, Phys. Rev. Lett. 112, 111103 (2014), arXiv:1402.6452 [gr-qc]. 